\title{
Réplique de James Iain Gow au compte rendu de son volume, Histoire de l'administration publique québécoise, 1867-1970, par Marc Vallières
}

\author{
James Iain Gow
}

Volume 41, numéro 1, été 1987

URI : https://id.erudit.org/iderudit/304544ar

DOI : https://doi.org/10.7202/304544ar

Aller au sommaire du numéro

Éditeur(s)

Institut d'histoire de l'Amérique française

ISSN

0035-2357 (imprimé)

1492-1383 (numérique)

Découvrir la revue

Citer ce document

Gow, J. I. (1987). Réplique de James Iain Gow au compte rendu de son volume, Histoire de l'administration publique québécoise, 1867-1970, par Marc Vallières. Revue d'histoire de l'Amérique française, 41(1), 111-112.

https://doi.org/10.7202/304544ar d'utilisation que vous pouvez consulter en ligne. 


\section{RÉPLIQUE DE JAMES IAIN GOW \\ AU COMPTE RENDU DE SON VOLUME, HISTOIRE DE L'ADMINISTRATION PUBLIQUE QUÉBÉCOISE, 1867-1970, PAR MARC VALLIERES}

J'accepte évidemment, les règles du jeu, l'auteur de la recension (40,3 (hiver 1987): 432-434), Marc Vallières, a droit à son opinion. Néanmoins, celle-ci est basée sur une lecture partielle aussi bien que partiale.

Monsieur Vallières me reproche l'ignorance de nombreux travaux et publications touchant des champs d'intervention étatique. C'est une critique que j'accepte, puisqu'il m'était impossible d'étudier en profondeur vingt-quatre domaines d'intervention étatique, sur une période de plus de cent ans. Par contre, il n'avait qu'à lire mon livre et pourtant, il laisse de côté des éléments essentiels à son propos. Il dit que le fait de retenir ensemble les interventions étatiques pour la période 1936-1970 m'empêche d'aborder de manière cohérente la période des années 1960 . Pourtant, j'ai tout un chapitre de plus de 70 
pages sur les années 1960 où j'essaie de cerner la nature de cet État technobureaucratique. Monsieur Vallières a dû le lire rapidement, car il me reproche d'avoir ignoré «totalement» le Conseil d'orientation économique, tandis qu'en réalité il est question de cet organisme pendant cinq pages (302-307). Également, dire comme le fait $\mathrm{M}$. Vallières que je n'ai présenté les changements de structure que de façon statique sous forme de quatre organigrammes risque de tromper le lecteur. D'une part, les raisons particulières de création des principales institutions sont données dans les chapitres traitant des interventions étatiques: il est alors question des raisons qui ont motivé la création du Conseil d'hygiène, le ministère des Affaires municipales, celui de l'Éducation, etc. Ensuite chaque organigramme est accompagné d'un développement accordant une attention particulière aux formes d'organisation privilégiées à chaque période.

S'il n'y avait que ces défauts de lecture dans cette recension, je pourrais me contenter de quelques réflexions sur la nature faillible des critiques aussi bien que des auteurs. Cependant, je trouve plus grave le fait que $\mathrm{M}$. Vallières ne dit mot sur mes hypothèses, ni sur mes conclusions. Puisque je suis politicologue et non historien de métier, il aurait été plus juste de sa part de reconnaître l'effort de formulation et de mise à l'essai de certaines hypothèses touchant la spécificité des interventions étatiques québécoises au Canada, l'influence de la croissance des missions étatiques sur l'administration, les liens entre les dépenses et les élections, la mise en place d'un système bureaucratique et l'émergence d'un pouvoir technocratique.

Il semble que M. Vallières ne s'intéresse qu'à l'un des deux volets de cette histoire, celui qui porte sur les interventions étatiques des gouvernements québécois pendant un siècle. Pourtant, celui-ci n'est abordé que pour être lié ensuite à l'autre volet, soit celui de l'évolution de l'administration proprement dite. Mise à part sa remarque trompeuse sur les organigrammes, la seule attention qu'il accorde à toutes ces questions administratives, pourtant l'objet premier du livre, est de reconnaître que «certains dossiers sont mieux traités, en particulier celui de la fonction publique». Monsieur Vallières dit que sa lecture du livre était décevante, il n'est pas le seul à en être déçu... 